\title{
Polarización artificial: cómo los discursos expresivos inflaman la percepción de polarización política en internet ${ }^{*}$
}

\author{
Artificial Polarisation: How Expressive Discourses Inflame \\ the Perception of Political Polarisation on the Internet
}

PEDRO JESÚS PÉREZ ZAFRILLA (Universitat de València)

Artículo recibido: 9 de septiembre de 2020

Solicitud de revisión: 12 de noviembre de 2020

Artículo aceptado: 14 de abril de 2021

Pérez Zafrilla, Pedro Jesús (2021). Polarización artificial: cómo los discursos expresivos inflaman la percepción de polarización política en internet. Recerca. Revista de Pensament $i$ Análisi, 26(2), pp. 1-23. doi: http://dx.doi.org/10.6035/recerca.4661

\section{Resumen}

En este trabajo analizo el fenómeno de la polarización política en internet. Argumento que el enfoque centrado en el filtro burbuja y las cámaras de eco tiene carencias. Para corregirlas, propongo el concepto de polarización artificial. Este concepto define el proceso por el que los usos expresivos de la comunicación, como discursos incendiarios o el exhibicionismo moral, provocan formas ficticias de polarización. Reconocer la polarización artificial permitirá comprender mejor los procesos de polarización en la red y sus efectos sobre el debate democrático.

Palabras clave: polarización artificial, polarización percibida, polarización de grupo, exhibicionismo moral.

\section{Abstract}

In this work I analyze the phenomenon of political polarization on the internet. I argue that the approach centered on the filter bubble and echo chambers has shortcomings. To solve them, I propose the concept of artificial polarization. This concept refers to the process by

\footnotetext{
Esta publicación se enmarca en el Proyecto de Investigación Científica y Desarrollo Tecnológico «Ética cordial y Democracia ante los retos de la Inteligencia Artificial» (PID2019-109078RB-C22), financiado por el Ministerio de Ciencia, Innovación y Universidades.
} 
which the expressive uses of communication, such as flaming or moral grandstanding, provoke fictitious forms of polarization. Recognizing the artificial polarization will allow a better understanding of the polarization processes in the network and their effects on the democratic debate.

Key Words: artificial polarization, perceived polarization, group polarization, moral grandstanding.

\section{INTRODUCCIÓN}

Las implicaciones de la inteligencia artificial sobre la democracia son un ámbito de estudio aun emergente que abarca diversos campos. Entre ellos sobresalen las propuestas de aplicación de algoritmos de inteligencia artificial para mejorar la toma de decisiones democráticas (Calvo, 2020). Pero también otros autores alertan de la amenaza que la inteligencia artificial representa para el debate democrático (Pariser, 2017). A este respecto, un tema que ha tomado especial vigor es el referido a la polarización política.

El estudio, teórico y empírico, de la polarización política en internet está focalizado en el consumo de contenido ideologizado y la interrelación de los individuos con sujetos afines ideológicamente. En el primer caso, las recomendaciones del algoritmo harían a los sujetos entrar en el filtro burbuja (Pariser, 2017), mientras que la interacción con afines daría lugar a la formación de cámaras de eco (Sunstein, 2003). Tanto las recomendaciones del algoritmo como la interacción con afines reforzarían a los sujetos en sus convicciones, produciendo una mayor división de la sociedad.

En este artículo pretendo defender que este enfoque del filtro burbuja y las cámaras de eco presenta limitaciones para comprender el fenómeno de la polarización en internet. Por un lado, porque los estudios realizados arrojan resultados contradictorios. Pero, además, porque ese enfoque no reconoce los usos expresivos que las personas hacen de internet. En ellos se produce una polarización artificial: un proceso de competencia de los sujetos por mejorar su estatus en la red mediante una escalada de expresiones emotivas y que da como resultado un clima de aparente polarización amplificado por la dinámica viralizante de la red. ${ }^{1}$ El concepto de polarización artificial permite abordar

\footnotetext{
El concepto polarización artificial está ya asentado en diferentes campos científicos, como la química o la física. En química se denomina polarización a la distribución desigual de la carga eléctrica en una molécula (molécula polar), frente a la molécula en la que esa distribución de la carga es simétrica (molécula apolar). La polarización artificial hace referencia al proceso por el que esa distribución desigual es inducida mediante la aplicación de un
} 
mejor a nivel académico la polarización política en internet y sus efectos sobre el debate democrático.

En primer lugar, presentaré las tesis de quienes defienden que el filtro burbuja y las cámaras de eco refuerzan la polarización de la sociedad y suponen una amenaza para la democracia. A continuación, expondré cómo los estudios empíricos sobre la incidencia de internet en la polarización política arrojan resultados contradictorios. Para reconducir el análisis de la polarización en la red de un modo adecuado, desarrollaré el concepto de polarización artificial, exponiendo sus supuestos psicológicos y filosóficos y desgranando sus aplicaciones concretas en los usos expresivos de la red.

\section{FILTRO BURBUJA Y CÁMARAS DE ECO}

El problema de la polarización política en internet tiene como referentes principales las reflexiones de Eli Pariser y Cass Sunstein. Ambos han expuesto los peligros que el filtro burbuja y las cámaras de eco tienen para el debate democrático, al generar procesos de polarización. Por ello, en esta primera sección presentaré los planteamientos de Pariser sobre el filtro burbuja y de Cass Sunstein sobre las cámaras de eco y la polarización grupal.

\subsection{El filtro burbuja}

Para críticos como Pariser (2017) la personalización del algoritmo arroja consecuencias nefastas en política. El hecho de que los buscadores devuelvan a los sujetos resultados acordes con sus perspectivas tiene el peligro de que los filtros les atrapen en búsquedas que refuercen sus creencias políticas y eliminen de los resultados perspectivas diferentes sobre esos temas. Pero si el algoritmo solo nos ofrece información que nos resulta agradable y nos oculta

campo electromagnético (Shalabi et al., 2007). En física, polarización artificial hace referencia a una forma de polarizar las ondas electromagnéticas. Se denomina polarización a la acción por la que las ondas electromagnéticas oscilan de una forma coherente, en lugar de propagarse en todas direcciones, como sucede de forma normal. La polarización se consigue mediante un elemento polarizador, que puede ser natural o artificial. La polarización natural sucede, por ejemplo, con el reflejo que provoca la superficie de la nieve. La polarización artificial designa la polarización provocada artificialmente, usando una lente, por ejemplo (Molino y Muñoz, 2019: 44). En cambio, yo empleo el concepto de polarización artificial para conceptualizar filosóficamente el proceso por el que diferentes usos expresivos de la comunicación, motivados por la búsqueda de estatus o el desahogo emocional — principalmente en internet—, forman en el público una falsa impresión de polarización política o de indignación sobre un tema. 
perspectivas disonantes, entonces el algoritmo está achicando nuestra percepción de la realidad y entorpece nuestra comprensión de los problemas. No nos mostrará una visión objetiva de los problemas, sino solo el lado coincidente con nuestra posición.

Además, la segmentación ideológica de los medios tradicionales guarda una diferencia clave con internet: mientras en un dial de radio los sujetos conocen qué otras emisoras hay y deciden no escuchar, en internet los sujetos no son conscientes de los contenidos que se están perdiendo porque solo tienen acceso a los resultados que les arroja el algoritmo, que van en sintonía con los intereses y las preferencias que los sujetos han expresado en sus búsquedas previas. Por lo tanto, los sujetos, debido al sesgo de confirmación, creen saber la verdad sobre un asunto - al haber encontrado contenidos que refuerzan sus creencias previas-, pero en realidad no son conscientes de toda la información disonante que el algoritmo les oculta. Esto hace que la calidad epistémica de la información que arrojan los algoritmos sea, cuando menos, cuestionable.

Pero el problema se agrava para Pariser con los agregadores de noticias. Estas plataformas estarían creando un universo informativo personalizado compuesto únicamente de noticias que resultan de interés para cada persona. Pero entonces esas personas tendrán la falsa impresión de que su limitado mundo de noticias es todo lo que existe, pudiendo permanecer ajenas a los problemas que son realmente importantes.

Por todo ello, el algoritmo representa un problema para el debate democrático. En primer lugar, porque hace que los ciudadanos cuenten con una limitada perspectiva sobre los problemas comunes, ya que el filtro burbuja opaca perspectivas diferentes que podrían ampliar su punto de vista. Así también, el filtro burbuja refuerza a cada grupo en sus creencias, con lo cual las personas acaban más alejadas en sus posiciones, generándose una polarización de la sociedad. Finalmente, que las personas formen sus distintas perspectivas y prioridades políticas en universos informativos paralelos hace que los temas que para unos ciudadanos son prioritarios, para otros puedan ser irrelevantes, ya que no aparecerán en sus pantallas. Esto rompe con la existencia de un espacio común en el que reconocer problemas compartidos a los que buscar una solución a través del dialogo, poniendo en riesgo la propia democracia.

\subsection{Las cámaras de eco}

Unido al filtro burbuja, otra gran amenaza para la democracia propiciada por la inteligencia artificial son las cámaras de eco. Afirma Sunstein que, aun- 
que internet facilita que gente con ideas diversas entre en contacto entre sí, la evidencia muestra que las personas no hacen sino replicar en la red la tendencia que tenemos los humanos de relacionarnos con gente de similares ideas, gustos e intereses. ${ }^{2}$ Concretamente, en cuestiones morales o políticas, las personas suelen emplear internet para contactar con gente que comparte una misma posición sobre ciertos asuntos o para buscar información que refuerce su punto de vista (Sunstein, 2003; 2008).

Ciertamente, antes de internet, la gente leía periódicos o veía canales de televisión que se ajustaban a su ideología. Sin embargo, internet permite personalizar la información a la que uno accede, de tal forma que puede elegir relacionarse solo con personas afines. Además, la persona puede bloquear aquella información que le resulte disonante. De esta forma, las personas acaban viviendo dentro de cámaras de eco (Sunstein, 2017). Podemos definir la cámara de eco como el «medio informativo cerrado que tiene el potencial de magnificar los mensajes que se transmiten dentro de él y aislar de ellos los mensajes disonantes» (Jamieson y Cappella, 2010: 76). Las redes sociales son un lugar propicio para generar cámaras de eco: no solo facilitan la conexión entre gente afín para discutir sobre los temas que les interesan, sino que, además, permiten aislar esa comunicación de contenidos disonantes, bloqueando a usuarios con planteamientos divergentes a los del grupo.

Ahora bien, para Sunstein (2017) las cámaras de eco conllevan dos amenazas fundamentales para la democracia. En primer lugar, al igual que vimos con los filtros burbuja, fomentan la fragmentación de la sociedad y dificultan el debate democrático. Pero mientras los filtros burbuja funcionan de forma opaca a los ciudadanos - mediante parámetros que estos desconocen, como denuncia Pariser-, las cámaras de eco son creadas por los propios sujetos al interactuar con gente de ideas comunes (Dutton y Fernández, 2018: 37). El segundo problema es que las cámaras de eco provocan una polarización de la sociedad. En concreto, las cámaras de eco generan lo que Sunstein denomina la polarización grupal.

La polarización grupal es el fenómeno por el cual, cuando los miembros de un grupo de gente de ideas afines hablan entre sí, el diálogo desplazará a los sujetos hacia una posición más extrema en la línea de lo que el grupo pensaba en el inicio del debate (Sunstein, 2002: 177; Sunstein, 2003). Aquí «posición más extrema» no debe entenderse en un sentido peyorativo de posición más

La mera existencia de los trolls refleja que la tendencia natural en internet es interactuar con los afines y permanecer ajeno a las posiciones contrarias. 
radical, sino como posición más convencida. Las causas de este proceso son tres.

La primera hace referencia a la información disponible. Cuando los miembros del grupo piensan relativamente igual sobre un tema, en la discusión habrá una cantidad desproporcionada de argumentos en la línea de la opinión mayoritaria del grupo, frente a los que pudieran escucharse en contra de esa medida. Eso hará que los sujetos que comparten esa posición mayoritaria se sientan más reforzados en sus ideas al escuchar que su punto de vista viene apoyado por argumentos nuevos que no conocían.

La segunda causa es la dinámica de influencia social: en un grupo los individuos desean mantener una imagen favorable ante los demás, así como tener una idea positiva de sí mismos (Schkade, Sunstein y Hastie, 2010: 242; Isenberg, 1986). Ello hace que los sujetos tiendan a presentar su posición sobre el tema de una forma que les haga ser bien percibidos por el resto (Sunstein, 2008: 92). Por ese motivo, cuando un grupo manifiesta una oposición hacia un tema, el control de armas, por ejemplo, aquellos que se oponen firmemente al control de armas se sentirán motivados a expresar su opinión. En cambio, aquellos que mantienen una opinión más moderada sobre el mismo tenderán a permanecer callados para no perjudicar su posición en el grupo. ${ }^{3}$

La tercera causa es la relación entre confianza, extremismo y corroboración: los individuos tienden a moverse en el diálogo en la dirección de los argumentos más persuasivos. Pero en un grupo de personas con ideas afines, los argumentos más persuasivos son los más vehementes y estos empujan al grupo hacia el extremo (Sunstein, 2002: 179). Esto hace que los miembros de ese grupo vean más confirmadas sus creencias previas y, por ende, se sientan más seguros de que mantienen la posición correcta sobre el asunto. En consecuencia, por el sesgo de confirmación, concederán una menor credibilidad a las ideas opuestas (Sunstein, 2017).

Así pues, la tesis de Pariser y Sunstein es que los fenómenos del filtro burbuja y las cámaras de eco pueden provocar una polarización de la sociedad. Al acceder solo a contenidos concordes con sus ideas, facilitados por el algoritmo, e interactuar en la red solo con personas afines, los sujetos acaban por radica-

\footnotetext{
Esta lógica fue denominada por Elisabeth Noelle-Neumann como la espiral del silencio. De acuerdo a esta teoría, los sujetos perciben intuitivamente en un grupo si su posición es o no dominante y, cuando advierten que es minoritaria, deciden no expresarla para mantener su reputación en el grupo. Antes de expresar que se pertenece a la minoría, los sujetos prefieren callarse y aparentar que apoyan la opinión más popular. Esto hace que, con el tiempo, los planteamientos que se perciben como minoritarios acaben por desaparecer de la esfera pública y solo se oigan los que gozan de un mayor prestigio social (Noelle-Neumann, 1995).
} 
lizar sus posiciones ideológicas, creando así una mayor separación entre los grupos que componen la sociedad y dificultando el debate democrático.

Ahora bien, estos procesos de polarización en la red tienen dos puntos en común: los sujetos hacen un uso de la razón comunicativa y cumplen las pretensiones de validez de la comunicación. Ambos elementos son, de hecho, las condiciones de posibilidad de la polarización. Los sujetos polarizan sus posiciones viendo contenido ideologizado porque presuponen la verdad de ese contenido y que responde a un interés de justicia - revelarles la maldad del adversario-. Del mismo modo, al dialogar con afines, los sujetos presuponen la sinceridad de sus interlocutores, la expectativa recíproca de convencimiento racional o la expectativa recíproca de estar guiados por un criterio de justicia (Cortina, 2007). Esta es una diferencia clave con la polarización artificial que presentaré después. Ahora abordaré las evidencias existentes en la bibliografía científica sobre la incidencia de los filtros burbuja y las cámaras de eco en la polarización.

\section{EVIDENCIAS SOBRE LA RELACIÓN ENTRE INTERNET Y LA POLARIZACIÓN}

Los estudios realizados sobre internet y polarización política arrojan datos contradictorios. Mientras unos trabajos apuntan a que internet fomenta la polarización de la sociedad, otros van en la dirección contraria, y su número es equivalente.

Los estudios que defienden que internet aumenta la polarización obtienen unas conclusiones bastante limitadas. Son, por lo general, ensayos sobre el comportamiento de los sujetos en una única plataforma, como YouTube (Cho, Ahmed, Hilbert, Liu y Luu, 2020) o Twitter (Basil et al., 2018; Hong y Kim, 2016; Gruzd y Roy, 2014), o una combinación de varias plataformas: buscadores, Facebook o agregadores de noticias (Flaxman, Goel y Rao, 2016). En concreto, miden los efectos que tiene la exposición de información política - confirmatoria o disonante-, facilitada por el algoritmo, sobre la polarización de los sujetos. ${ }^{4}$ La exposición a información política disonante se emplea

\footnotetext{
Existen en ciencia política dos procedimientos para evaluar la polarización política: la polarización ideológica y la polarización afectiva. La polarización ideológica tiene una base cognitiva: analiza la división de creencias y opiniones de los distintos grupos ideológicos sobre cuestiones políticas. Por su parte, la polarización afectiva analiza el nivel que alcanzan los sentimientos negativos de rechazo o animadversión de los sujetos hacia sus adversarios ideológicos (Pérez Zafrilla, 2020).
} 
para medir el grado de polarización que provoca la visualización de ese contenido. La exposición a información afín permite a esos estudios corroborar las mecánicas del algoritmo señaladas por Pariser: el algoritmo ofrece a los sujetos contenidos acordes a sus creencias, en función del perfil que sobre ellos ha realizado a partir de los términos introducidos por los individuos. Esta recomendación de contenidos confirmatorios provoca que los sujetos refuercen sus opiniones, haciendo sus creencias y actitudes más rígidas, produciéndose una polarización de sus posiciones, como explica Sunstein. Ahora bien, los estudios rechazan concluir que exista un determinismo tecnológico por el que el algoritmo encierre a los sujetos en filtros burbuja donde solo acaben accediendo a contenido afín e ignorando las informaciones disonantes. También, frente a lo defendido por Pariser, los agregadores de noticias, lejos de encerrar a los sujetos en un filtro burbuja, proporcionan una variedad de fuentes informativas (Flaxman, Goel y Rao, 2016: 312).

En el lado opuesto están los autores que rechazan que internet y, especialmente, las redes sociales sean causantes de la polarización política. Sus argumentos son principalmente tres.

El primero es una crítica metodológica a los estudios que pretenden mostrar el efecto causal de internet en la polarización. Esos estudios están basados en dos fallos metodológicos (Barberá, 2014: 5; Dubois y Blank, 2018: 732). El primero es que analizan el comportamiento aislado de los sujetos en una única plataforma. Pero esos estudios no serían representativos del comportamiento de los sujetos en internet para informarse de política, ya que la dieta informativa de los sujetos en internet proviene de varias vías: consulta directa de webs, redes sociales, búsqueda de contenidos en Google o agregadores de noticias. Por ese motivo, para saber si internet aumenta la polarización, hay que evaluar el comportamiento de los sujetos en el conjunto de plataformas. El segundo fallo metodológico es que esos estudios se centran en analizar la autoexposición explícita a contenido político, cuando esa es solo una pequeña parte de la información política que los sujetos reciben. Los sujetos reciben información política también de forma implícita a través de las noticias que publican sus contactos en sus redes sociales, y mucha de esa información puede ser disonante (Zuiderveen Borgesius et al., 2016: 8).

Un segundo argumento tiene que ver con la plataforma empleada por los sujetos. Ciertamente, hay formatos, como los foros o los blogs, que pueden generar cámaras de eco, ya que los usuarios acceden a ellos buscando un contenido específico para reforzar sus convicciones sobre ciertos asuntos (Lawrence, Sides y Farrell, 2010). De hecho, el propio Sunstein asienta su tesis 
principalmente en datos referidos a blogs (Sunstein, 2003). Sin embargo, las redes sociales, lejos de fomentar la polarización, permiten a los sujetos acceder a contenidos disonantes gracias a las noticias que cuelgan sus amigos, compañeros de trabajo o familiares (Beam, Hutchens y Hmielowski, 2018: 950). Así lo reflejan estudios como el de Barberá (2014: 17): aunque en redes sociales los sujetos siguen principalmente a gente afín, la heterogeneidad de las redes es mayor de la que se piensa - siendo mayor ente los sujetos más moderados-. En España cada usuario de Twitter tiene de media un $45 \%$ de contactos -entre seguidores y aquellos a los que sigue-que no comparten la ideología de ese usuario; en Alemania ese porcentaje es del $4 \%$ y en EE. UU., del $33 \%$.

Otros autores van más allá y señalan que las cámaras de eco y el filtro burbuja son fenómenos sobrevalorados por las implicaciones políticas que se les atribuyen, pero cuya incidencia en la actualidad es poco significativa. Prueba de ello sería que, como acabamos de decir, los sujetos emplean diferentes canales para informarse online (Dubois y Blank, 2018: 739). Pero, además, el uso activo de la red (Groshek y Koc-Michalska, 2017: 1397) y el mayor interés por la política se correlacionan con la consulta de una mayor variedad de fuentes de información, así como con una mayor heterogeneidad en las interacciones en redes sociales. Los sujetos más vulnerables a caer en cámaras de eco resultan ser los menos interesados en política y con menos habilidades digitales, pero su porcentaje es bajo, en torno al $1 \%$ en EE. UU. y el $4 \%$ en Italia (Dutton y Fernández, 2018: 39).

Como vemos, los estudios sobre la incidencia de internet en la polarización arrojan resultados contradictorios. Esto es debido a que los defensores de que internet fomenta la polarización y quienes rebaten esa tesis abordan cuestiones distintas. Los primeros analizan el funcionamiento del algoritmo en una plataforma concreta y su efecto sobre la polarización de los sujetos. Mientras, los segundos se centran en rechazar la idea de que el algoritmo pueda encerrar a los sujetos en el filtro burbuja, porque los individuos emplean diversas plataformas a través de las que reciben información disonante.

Pero, a pesar de su diferente enfoque metodológico, todos estos estudios comparten dos errores fundamentales.

El primero es que se centran en demostrar si las plataformas en sí mismas fomentan o no la polarización, pero no atienden al uso que los usuarios hacen de ellas, más allá del uso activo o pasivo de la red (Groshek y Koc-Michalska, 2017: 1397). Este es un punto advertido por algunos autores. Barberá (2014: 20) reconoce que puede haber personas con una fuerte ideología que decidan crear una cuenta anónima en Twitter para seguir solo a perfiles que comparten su 
ideología, algo que su estudio no tiene en cuenta, por lo que su estudio estaría sobreestimando la heterogeneidad de los perfiles en Twitter. Así, también, Cho, Ahmed, Hilbert, Liu y Luu (2020: 17) reconocen que las recomendaciones del algoritmo son fruto de los términos introducidos por los sujetos. Por lo tanto, el algoritmo contribuirá a reforzar las convicciones de la persona en función de los términos que esta decida introducir, y eso dependerá del uso - polarizante o no- que quiera hacer de esa plataforma. Así pues, el análisis de la polarización en internet no debe centrarse en si el algoritmo fomenta la polarización en una u otra plataforma, sino en el uso que los sujetos hacen de ella.

En segundo lugar, y fundamentalmente, el hecho de que los estudios no atiendan a los distintos usos que la gente hace de internet implica que estos estudios no discriminan entre aquellos usos que fomentan una polarización real entre los usuarios y cuándo se produce una polarización artificial: esto es, aquellos usos puramente expresivos de la comunicación —en la red-con los que el sujeto busca mejorar su posición en el grupo y que generan una falsa percepción de polarización o indignación. La polarización artificial se puede producir tanto por un uso pasivo de la red - la visualización de contenidos altamente ideológicos-, como por usos activos - la participación en linchamientos virtuales o el exhibicionismo moral-.

\section{BASES DE LA POLARIZACIÓN ARTIFICIAL}

La idea de polarización artificial permite conceptualizar filosóficamente el proceso que da lugar a un fenómeno conocido en la ciencia política: la polarización percibida. Esta consiste en la tendencia de los sujetos a sobreestimar el grado de polarización que hay entre su grupo ideológico y sus adversarios. A pesar de que las personas tienen posiciones relativamente moderadas, muchas piensan que sus adversarios ideológicos tienen actitudes más extremas de las que realmente tienen y que, por lo tanto, la brecha entre ellos es mayor de la que existe en realidad (Kim, 2016: 23). Es decir, la polarización percibida se produce cuando existe una disonancia entre la percepción de la realidad política por parte del electorado - la impresión de que los adversarios son muy radicales- y cómo es el electorado en sí. La polarización percibida es, por tanto, una falsa percepción de polarización. 
Las causas señaladas de la polarización percibida son dos. La primera tiene que ver con el consumo de contenido ideologizado, tanto online como offline. En un espacio audiovisual fragmentado por los canales de televisión por cable e internet, los programas, para atraer a la audiencia, tienden a radicalizar sus contenidos y enfatizar los desacuerdos entre los grupos. Además, en los medios rige la simplificación de los problemas presentando dos opciones únicas entre las que se debe optar. Esto permite a los medios formar una narrativa que divide a la sociedad en buenos y malos (Yang et al., 2016: 353). A esto se une que los medios partidistas y los canales de internet ofrecen a su audiencia estereotipos negativos del adversario ideológico con el objetivo de presentar sus posiciones como extremas o ridículas.

Los estudios reflejan que tanto los medios de comunicación como internet fomentan la polarización percibida (Levenduski y Malhotra, 2016: 388). Es más, un estudio apunta a que internet produce una mayor polarización percibida que los medios tradicionales (Yang et al., 2016: 359). Ahora bien, si internet genera una mayor polarización percibida que los medios tradicionales, ¿produce también una mayor polarización de la sociedad? Estudios realizados tanto en EE. UU. (Boxell, Gentzkow y Shapiro, 2017: 10615; Hwang, Kim y Hug, 2014: 629) como en diez países (Yang et al., 2016) no encontraron una relación directa entre polarización percibida y polarización real de los sujetos. Todos coinciden en que internet aumenta en los sujetos - especialmente entre los jóvenes - la polarización percibida en mayor medida que la polarización real -afectiva o ideológica-. Esto indicaría que la gente que se informa de política por internet percibe una política más polarizada, pero la información offline polariza más que la online.

La otra causa de la polarización percibida hace referencia a los sesgos provocados por la visualización de cierto contenido político. El primero es la heurística de disponibilidad (Yang et al., 2016: 354): los sujetos, al formarse sus juicios sobre cómo son y qué piensan sus adversarios ideológicos, echan mano de esos estereotipos creados por su universo informativo. El segundo sesgo es el de intragrupo/exagrupo (Clark y Winegard, 2020: 2-3; Hwang, Kim y Huh, 2014: 629): Los neurocientíficos defienden que tenemos una naturaleza tribal que nos predispone a evaluar positivamente a los miembros y las posiciones de nuestro grupo y negativamente las posiciones y los miembros del grupo contrario. Por ello, la exposición a unos contenidos que enfatizan el conflicto y presentan a los adversarios ideológicos como una amenaza reforzará en la au- 
diencia una imagen negativa de los adversarios, alejada de cómo son estos en realidad.

Pues bien, el fenómeno de la polarización percibida, producido por ese consumo -online u offline - de contenidos ideologizados - como discursos incendiarios-, responde a una misma lógica que se da también en unos usos activos de la red: los linchamientos virtuales o el exhibicionismo moral, cuando tiene lugar entre gente afín. Aunque algunos autores asocian estos usos activos de la red con formas reales de polarización (Grubbs et al., 2019: 31; Johnen, Jungblut y Ziegele, 2017: 3143; Haidt y Lukianoff, 2019: 218), yo defiendo que estos usos desatan en realidad una dinámica de polarización artificial que produce una falsa impresión de polarización. Ello es así porque todos estos usos comparten tres aspectos: los sujetos actúan desde su identidad de grupo, hacen un uso expresivo de la red con el fin de mejorar su estatus en el grupo y la viralización de esos contenidos expresivos crea en la audiencia una imagen deformada del adversario. Estos tres elementos combinados potencian la percepción errónea de polarización —o indignación- en internet.

\section{POLARIZACIÓN ARTIFICIAL}

Si asumimos la tesis de que somos seres tribales, en aquellos contextos en que los sujetos actúan guiados por su identidad grupal se activa en ellos la capacidad de trascender el interés propio y de pensar y sentir como miembros del grupo (Haidt, 2019). Esta adopción de la identidad grupal permitirá a los sujetos ver la realidad con las lentes del grupo.

La adopción de la identidad grupal sucede en internet en ciertos usos que hacen las personas de sus redes sociales, como, por ejemplo, cuando crean una cuenta anónima en Twitter para seguir otros perfiles afines. Esa desindividuación permite al sujeto identificarse más con las normas del grupo y sentir como los miembros del grupo (Haidt y Lukianoff, 2019). Pero especialmente, como defienden Brady, Crockett y Van Bavel (2020: 981) con su modelo del contagio moral, la identidad grupal se refuerza en internet por la visualización de contenidos emocionales que son expresados con emociones morales. Afirman, además, que el contenido expresado con emociones morales de indignación, ira o culpa tiene una mayor viralización en internet. El motivo es que los algoritmos de Google y Facebook tienden a hacer los contenidos indignantes más visibles al atraer más la atención de los usuarios. En este sentido, el algoritmo de estas plataformas estaría alineado con nuestras tendencias evolutivas: 
la muestra de ira e indignación y la condena moral de los adversarios en la red - principalmente mediante un lenguaje agresivo que incluye términos relativos a emociones morales- despierta en los miembros del grupo emociones morales, al percibir los sujetos que su identidad de grupo está siendo amenazada. Estas emociones les empujan a sumarse a esa condena de un acto que se percibe como inmoral, en virtud de la tendencia evolutiva de condenar al infractor de las normas del grupo. Porque la adopción de la identidad de grupo lleva a los sujetos a actuar en la red para mantener la imagen positiva del grupo frente a los adversarios.

Otra tendencia evolutiva causante de la polarización artificial es el deseo de los sujetos de mejorar su reputación dentro del grupo. Según Mercier y Sperber (2011), esta motivación explicaría el origen del razonamiento. Este no evolucionó porque nos permitiera conocer la realidad, sino porque ayudaba a los sujetos a mejorar su estatus en el grupo proyectando una buena imagen ante los demás. Para Badry, Crockett y Van Bavel (2020: 989-990), el modo que tendrán los sujetos de aumentar su reputación en el grupo será expresar emociones de indignación hacia los adversarios. A ello contribuyen características de las redes sociales, como los likes, los seguidores o los retuits. Estos elementos suponen una recompensa para los sujetos que publican contenidos que refuerzan la posición del grupo, que, a la vez, impulsan a los sujetos a publicar contenidos más radicales. Esta tendencia a la radicalidad viene fomentada por otro elemento causante de la polarización artificial en la red: el uso expresivo de las comunicaciones.

Es un lugar común en la bibliografía sobre internet que la democratización del debate público, lejos de fomentar el dialogo razonado, ha traído más bien una cacofonía digital (Han, 2014; Arias Maldonado, 2016). Esto explica que en las redes prime la comunicación expresiva sobre el diálogo racional. Por un lado, la gente emplea la red para expresar opiniones, sentimientos y estados de ánimo - especialmente la indignación, como señalaba arriba-, y lo hace con mensajes emocionalmente cargados que atraen la atención de otros afines. Por otro, los sujetos se mueven contagiados por los estímulos emocionales recibidos, produciéndose así la conectividad por la que los individuos se adhieren de forma instantánea a corrientes de opinión promovidas por su tribu moral.

Ahora bien, esta primacía de la emotividad y este uso expresivo de la red potencian que los sujetos no se guíen por la razón comunicativa sino por la estratégica: dado que en estos casos los individuos no dialogan, sino que expresan opiniones cargadas emotivamente por mero desahogo o en busca de reco- 
nocimiento, muchos sujetos no dicen lo que realmente piensan, sino lo que les permite aumentar su reputación en la red. Del mismo modo, no están orientados al entendimiento y al acuerdo, sino a la mera obtención de prestigio dentro del grupo. Para ello, cualquier medio será válido, desde la mentira al insulto. Además, los juicios no expresan creencias sino emociones. Por eso precisamente se generan así contextos comunicativos en los que se produce una polarización artificial pero no una polarización real.

Así, el concepto de polarización artificial designa el proceso que da lugar a un clima de aparente polarización o indignación en la red, a partir de un conjunto de usos de internet que comparten una serie de supuestos psicológicos y filosóficos. Los supuestos psicológicos son: la adopción de la identidad de grupo, el refuerzo del sesgo intragrupo/extragrupo y la motivación de mejorar la posición en el grupo mediante el estímulo de la indignación moral en la audiencia. Los supuestos filosóficos son: el uso de la razón estratégica, el uso expresivo del lenguaje, la ausencia de diálogo y la quiebra de las pretensiones de validez de la comunicación.

La polarización artificial se compone de dos elementos. Por un lado, el discurso emotivo que hace el sujeto para reclamar la atención y que activa en su audiencia el sesgo intragrupo/extragrupo. Por otro, el proceso de contagio emocional desencadenado por esa expresión y que lleva al resto de sujetos a unirse a ese discurso como forma de manifestar su adhesión al grupo. A continuación, expondré los principales ejemplos.

En primer lugar, tenemos los discursos incendiarios que ciertos personajes -tuiteros, youtubers, blogueros - publican contra sus adversarios ideológicos. Son contenidos puramente hiperbólicos, trufados de expresiones con emociones morales. En ellos se hacen retratos hirientes del adversario, deshumanizándolo y presentándolo como una amenaza existencial. Se diferencian de los discursos de odio por tener una diferente finalidad. Mientras los discursos de odio pretenden incitar el odio contra determinados colectivos (Cortina, 2017), los discursos incendiarios tienen una naturaleza puramente ficcional, aunque sus consecuencias sean similares en el plano político. Los discursos incendiarios se definen por la motivación que impulsa a sus creadores: obtener notoriedad en la red explotando en su audiencia los sentimientos de indignación moral y los sesgos cognitivos de confirmación y de intragrupo/exagrupo. Así, consiguen reforzar emocionalmente la identidad grupal de sus seguidores y una imagen negativa del adversario. Pero también tratan de provocar la reacción airada de sus adversarios. Ello les permite aumentar la viralidad de sus menajes. 
Cuando estos personajes publican contenidos incendiarios se crea en la audiencia un clima de hostilidad que hace parecer que el nivel de polarización social es enorme. Ello es así porque, como dije antes, los mensajes cargados emotivamente provocan en sus receptores un sentimiento de ira hacia los adversarios, al percibir amenazados los valores del grupo. Sin embargo, esa oposición visceral mostrada en la red por mero interés reputacional refuerza la impresión negativa que la audiencia tiene del adversario - polarización percibida-, creando una imagen del adversario alejada de cómo es este en realidad (Brady, Crockett y Van Bavel, 2020: 989). Por eso las redes inflaman una confrontación alejada de la oposición existente en la sociedad. Así, el discurso incendiario produce en realidad una polarización artificial: crea un clima de aparente polarización por un uso de la comunicación que busca meramente mejorar el estatus del autor en su grupo mediante la condena visceral del adversario.

Podría objetarse que, quizá, el autor del discurso incendiario crea realmente lo que afirma. Pero, con independencia de ello, lo que define la polarización artificial es que la intención del sujeto al expresar ese discurso es mejorar su estatus en el grupo, excitando la indignación en su audiencia. Este es el punto nuclear de la polarización artificial. Como acertadamente señala Adela Cortina recordando la distinción aristotélica entre voz y palabra, la palabra se encarna en un diálogo y presupone las pretensiones de validez que hacen posible la comunicación (Cortina, 2021). Pero el discurso incendiario no busca iniciar un diálogo. Además, socava las pretensiones de verdad, veracidad y justicia, pues hace un retrato deformado de la realidad y la intención de su autor no es expresar verdad ni convencer racionalmente, sino obtener notoriedad manipulando emociones. En este sentido, la polarización artificial arranca de esta quiebra de las condiciones que posibilitan la comunicación e inicia un proceso que entorpece el discurso público. Porque buscar notoriedad explotando la indignación y fomentando una imagen destructiva del adversario impide el diálogo y, en consecuencia, deforma la palabra, degradándola a una voz que llama a la unión tribal.

De ahí que el contagio emocional producido por el discurso incendiario tampoco deba confundirse con una polarización real de los sujetos en sus posiciones. Porque, como señalé antes, la polarización de posiciones presupone el uso de la razón comunicativa y aceptar las pretensiones de validez del lenguaje. En cambio, la polarización artificial produce un reforzamiento del sentimiento de la identidad grupal - y un falso clima de indignación - provocado por 
una agregación de mensajes que meramente buscan obtener notoriedad en respuesta a un discurso inicial emotivamente cargado.

Por todo ello, es necesario denunciar el carácter artificial de esta polarización - consistente en generar una peor imagen del adversario mediante un uso expresivo de la red-. Así se podrá evitar que quienes no reconocen la naturaleza puramente retórica de esos contenidos puedan interpretarlos desde una razón comunicativa, atribuirles unas pretensiones de validez que no cumplen $\mathrm{y}$, con ello, polarizar sus posiciones al visualizarlos.

Un segundo ejemplo es el exhibicionismo moral: las expresiones de indignación moral que tienen como objetivo obtener el reconocimiento de la audiencia a su especial sensibilidad ética (Tosi y Warmke, 2016: 199). Cuando este fenómeno tiene lugar en un ambiente plural, la reacción típica de la audiencia es el rechazo hacia el exhibicionista moral, al no atribuir a este una pretensión de veracidad por su uso expresivo del lenguaje. Sin embargo, cuando el exhibicionismo se produce entre personas afines, especialmente en la red, se desata la dinámica propia de la polarización artificial.

La exhibición de indignación - sea o no sincera- por la violación de los valores del grupo, hecha por la persona con el objetivo de recabar atención en la red apelando a emociones morales, activa inconscientemente en su audiencia afín el sentimiento de identidad de grupo y el rechazo al infractor. Entonces los contactos que forman parte de ese grupo, al recibir el impacto emocional de ese mensaje, se verán forzados a posicionarse sobre ese acto como forma de reafirmar su identidad de grupo. Además, entrarán en una escalada condenatoria para mejorar su posición en el grupo. Esto les llevará a sumarse a esa denuncia y a realizar una condena más dura que quienes le precedieron en el muro. Llegado a un cierto clímax en esa escalada condenatoria, el resto de intervinientes se sumará a la posición más punitiva, añadiendo nuevos matices que refuercen su posición en el grupo, aunque queden por debajo del que hizo la afirmación más severa.

A diferencia de la vida real, donde las denuncias o condenas pueden conllevar la respuesta del afectado, en el mundo virtual la condena no tiene apenas riesgos y, en cambio, reporta el beneficio de mejorar la posición en el grupo. Además, en la red la condena se puede hacer de manera inmediata y como respuesta a la sensación de indignación sufrida al recibir los mensajes. Todo ello hace que el sujeto no evalúe la situación ni dé una respuesta proporcionada a la gravedad del asunto. Simplemente expresa una opinión gratuita que sabe que le hará quedar bien ante los demás. De hecho, la escalada condenatoria puede llevar a los sujetos a decir cosas que ni siquiera piensan. Pero las 
expresan simplemente porque mejorarán su posición en el grupo y porque les genera un desahogo emocional. En consecuencia, la escalada condenatoria no responde a una indignación real por un hecho, sino a un deseo de mantener su estatus en el grupo y de expresar un sentimiento de indignación (Grubbs et al., 2019: 3). Dicho de otra forma, el exhibicionismo moral se basa en una burbuja artificial de indignación inflamada por la dinámica emotiva y viralizante de las redes.

Por ese motivo, aunque los teóricos del exhibicionismo moral relacionan ese fenómeno con la polarización de grupo descrita por Sunstein (Tosi y Warmke, 2016: 206-208), en mi opinión el exhibicionismo moral posee cuatro rasgos que lo alejan de la polarización grupal de Sunstein y lo convierten en un exponente de la polarización artificial.

El primero es el tipo de comunicación empleada: en la polarización artificial el uso de la comunicación es puramente emotivo y estratégico. La polarización artificial se compone de una sucesión de opiniones expresadas afectivamente en la red en busca de la aprobación de los otros. Pero los sujetos no dialogan. Solo se prestan atención para superar en intensidad a su predecesor. Se participa en un exhibicionismo moral no necesariamente porque se esté indignado por el hecho que se critica, sino para dar expresión a un sentimiento de indignación contagiado por la exposición al contenido recibido o para mantener la reputación dentro del grupo. Por ese motivo, si para ascender en estatus hay que recurrir a la exageración o a la mentira, se hace sin el menor reparo.

En cambio, en la polarización grupal de Sunstein los sujetos dialogan desde la razón comunicativa y respetando el deber rawlsiano de la civilidad: se escuchan, argumentan sinceramente, evalúan la posición del otro y conforman su opinión a la luz de los argumentos escuchados — que van todos en la misma dirección y, por ese motivo, polarizan la posición del grupo-.

Ciertamente, la polarización de grupo tiene en común con la polarización artificial el factor de la comparación social. Pero justo en este punto surge una segunda diferencia entre ambas: en la polarización de grupo la presión social - por el respeto a las normas del discurso y por el efecto de la espiral del silencio- lleva a los más moderados a callar para no empeorar su posición en el grupo. Por el contrario, en la polarización artificial la presión social es desinhibidora: el uso expresivo de la red incita a los miembros del grupo a hacer declaraciones inflamantes e insinceras para ganar reputación. Esto nos conecta con el tercer elemento. 
En la polarización artificial la escalada condenatoria y la lucha por mejorar la posición dentro del grupo es una resultante del propio uso emotivo y estratégico que los sujetos hacen de las redes. Todos pueden expresar opiniones insinceras porque su objetivo es mejorar su reputación. Sin embargo, en la polarización grupal de Sunstein no hay una pugna entre los individuos por mejorar su estatus. Los sujetos simplemente son convencidos por quien expone el argumento más persuasivo en la línea de lo que todos piensan. La única escalada que se produce es en la firmeza con la que los sujetos mantienen sus posiciones al final del diálogo.

El cuarto elemento es relativo a la polarización: en la polarización de grupo los sujetos polarizan sus posiciones porque pasan a mantener sus ideas previas de una forma más firme a la luz de nuevos argumentos. En cambio, en el exhibicionismo moral no hay una polarización real de posiciones, sino una simple competición por obtener notoriedad. En el exhibicionismo moral hay una polarización artificial en el sentido aquí expuesto.

Finalmente, esta polarización artificial se produce también en los linchamientos virtuales. Muchos sujetos que se suman a esa acción no lo hacen porque tengan un conocimiento del caso que les lleve a indignarse y mostrar su rechazo a esa persona. De hecho, internet permite unirse a un linchamiento a personas que viven en otros países y nada conocen del caso, contribuyendo así a su viralización. Estudios reflejan que estas personas se unen al linchamiento guiadas por un sentido de pertenencia grupal y como una forma de mejorar su estatus dentro del grupo (Johnen, Jungblut y Ziegele, 2017: 3144; Haidt y Lukianoff, 2019: 115). Rasgos de este fenómeno, como su volatilidad, la desproporcionalidad entre la acusación realizada y las consecuencias del linchamiento y, principalmente, la falsa percepción de consenso - al anular cualquier diferencia de opinión-, muestran que los sujetos actúan para mostrar su adhesión al grupo en respuesta a una indignación moral inflamada por la red, pero que las personas en realidad no comparten - al no conocer el caso-, y por un mero afán de reputación. Por eso mantengo que el linchamiento virtual no es una muestra de polarización, sino que reproduce los elementos de la polarización artificial: hay una manifestación expresiva de condena para acaparar atención - que activa la identidad grupal y la imagen negativa del infractor - y una consiguiente adhesión emocional - y estratégica- de la audiencia como forma de afirmar la identidad grupal.

Así pues, podemos comprobar cómo el uso de la razón estratégica y las formas expresivas de comunicación en la red generan una polarización artificial distinta de la polarización real, ya que esta presupone el empleo de la ra- 
zón comunicativa. Por ese motivo, el estudio de la polarización en internet debe tener en cuenta el fenómeno de la polarización artificial y discriminar los diferentes usos que los sujetos hacen de la comunicación en la red.

\section{CONCLUSIÓN}

En este artículo he presentado la idea de la polarización artificial. Este concepto puede clarificar el fenómeno de la polarización en internet, tanto en el ámbito académico como social y político.

En el ámbito académico, en primer lugar, la polarización artificial articula una conceptualización de las dinámicas que generan formas de polarización percibida en la red y permite diferenciar esa polarización percibida de los procesos de polarización real. Así, también, demuestra que el análisis de la polarización en internet no puede realizarse sin tener en cuenta los usos que los sujetos hacen de la red. Sobre esta base, los estudios podrán discriminar entre aquellas situaciones que reflejen una polarización real frente a los estudios basados en burbujas de polarización inducidas por los usos expresivos de la red. De esta forma se podrá analizar de manera real el problema de la polarización en internet, conociendo su verdadera dimensión y complejidad.

En el ámbito social y político, reconocer la polarización artificial arroja múltiples ventajas. Permite que los ciudadanos sean conscientes de cómo en los contextos expresivos se produce una polarización ficticia que no deben fomentar, al venir causada por una competencia por la notoriedad. Además, ayuda a desenmascarar los discursos incendiarios, al delatar su naturaleza puramente hiperbólica. Si los ciudadanos muestran lucidez al reconocer esas burbujas de polarización y no se dejan arrastrar por ellas, las consecuencias políticas serán enormes: habrá una mejor comprensión del adversario político, será más fácil llegar a acuerdos y la democracia saldrá fortalecida.

\section{BIBLIOGRAFÍA}

Arias Maldonado, Manuel (2016). La democracia sentimental. Política y emociones en el siglo XXI. Barcelona: Página indómita.

Barberá, Pablo (2014). How social media reduces mass political polarization. Evidence from Germany, Spain and the US. Recuperado de: 
http://pablobarbera.com/static/barbera_polarization_APSA.pdf [Consultado el 28 de mayo de 2020].

Basil, Christopher A., Argyle, Lisa P., Brown, Taylor W., Bumpus, John P., Chen, Haohan, Hunzaker, M.B. Fallin, Lee, Jaemin, Mann Marcus, Merhout, Frieden y Volfovsky, Alexander (2018). Exposure to opposing views on social media can increase political polarization. PNAS, 15, 9216-9221.

Beam, Michael, Hutchens, Myiah y Hmielowski, Jay (2018). Facebook news and (de)polarization: reinforcing spirals in the 2016 US elections. Information, communication and society, 21, 940-958.

Boxell, Levi, Gentzkow, Matthew y Shapiro, Jesse M. (2017). Greater internet use is not associated with faster growth in political polarization among US demographic groups. PNAS, 114, 10.612-10.617.

Brady, William, Crockett, Molly J. y Van Bavel, Jay (2020). The MAD Model of Moral Contagion: The role of Motivation, Attention and Design in the spread of moralized content online. Perspectives on Psychological Science, 15, 978-1010.

Calvo, Patrici (2020). Democracia aumentada: un ecosistema cibernético para una participación política basada en algoritmos. Ápeiron. Estudios de filosofía, 12, 129-141.

Cho, Jaeho, Ahmed, Saifuddin, Hilbert, Martin, Liu, Billy y Luu, Jonathan (2020). Do search algorithms endanger democracy? An experimental investigation of algorithm effects on political polarization. Journal of Broadcasting \& Electronic Media, 64, 150-172.

Clark, Cory y Winegard, Bo. (2020). Tribalism in War and Peace: the nature and evolution of ideological epistemology and its significance for modern social science. Psychological Inquiry, 31, 1-22.

Cortina, Adela (2007). Ética de la razón cordial. Oviedo: Nobel.

Cortina, Adela (2017). Aporofobia, el rechazo al pobre. Barcelona: Paidós. Cortina, Adela (2021). Ética cosmopolita. una apuesta por la cordura en tiempos de pandemia. Barcelona: Paidós. 
Dubois, Elizabeth y Blank, Grant (2018). The echo chamber is overstated: the moderating effect of political interest and diverse media. Information, Communication \& Society, 21, 729-745.

Dutton, William y Fernández, Laleah (2018). How susceptible are internet users? InterMEDIA, $46,36-40$.

Flaxman, Seth, Goel, Sharad y Rao, Justin M. (2016). Filter bubbles, echo chambers and online news consumption. Public Opinion Quarterly, 8o, 298-320.

Groshek, Jacob y Koc-Michalska, Karolina (2017). Helping populism win? Social media use, filter bubbles, and support for populist presidential candidates in the 2016 US election campaign. Information, Communication and Society, 20, 9, 1389-1407.

Grubbs, Joshua B., Warmke, Brandon, Tosi, Justin, James, Shanti y Campbell, Keith (2019). Moral grandstanding in public discourse: Status-seeking motives as a potential explanatory mechanism in predicting conflict. PLOS ONE, 14(10), eo223749.

Gruzd, Anatoly y Roy, Jeffrey (2014). Investigating political polarization on Twitter: a Canadian perspective. Policy \& Internet, 6, 2845 .

Haidt, Jonathan (2019). La mente de los justos. Barcelona: Deusto.

Haidt, Jonathan y Lukianoff, Greg (2019). La transformación de la mente moderna. Barcelona: Deusto.

Han, Byung-Chul (2014). En el enjambre. Barcelona: Herder.

Hong, Sounman y Kim, Sun H. (2016). Political polarization on twitter: Implications for the use of social media in digital governments. Government Information Quarterly, 33, 777-782.

Hwang, Hyunseo, Kim, Youngju y Huh, Catherine (2014). Seeing is Believing: Effects of Uncivil Online Debate on Political Polarization and Expectations of Deliberation. Journal of Broadcasting \& Electronic Media, 58, 621-633.

Isenberg, Daniel (1986). Group polarization: a critical review and metaanalysis. Journal of Social and Personal Psychology, 50, 1141-1151. 
Jamieson, Kathleen H. y Cappella, Joseph N. (2010). Echo chamber: Rush Limbaugh and the conservative media establishment. Oxford: Oxford University Press.

Johnen, Marius, Jungblut, Marc y Ziegele, Marc (2017). The digital outcry: What incites participation behavior in an online firestorm? New Media \& Society, 20, 3140-3160.

Kim, Youngju (2016). How do news frames influence mass political polarization? Tesis doctoral. Tuscaloosa: University of Alabama.

Lawrence, Eric, Sides, John y Farrell, Henry (2010). Self segregation or deliberation? Blog relationships, participation and polarization in American politics. Perspectives in Politics, 8, 141-157.

Levendusky, Matthew y Malhotra, Neil (2016). Misperceptions of partisan polarization in the American public. Public Opinion Quarterly, 8o, 378-391.

Mercier, Hugo y Sperber, Dan (2011). Why do humans reason? Arguments for an argumentative theory of reasoning. Behavioral and Brain Sciences, 34, 57-111.

Molino, Fernando del y Muñoz, Adolfo (2019). Polarization mapping. Computers \& Graphics, 83, 42-50.

Noelle-Neumann, Elisabeth (1995). La espiral del silencio. Opinión pública: nuestra piel social. Barcelona: Paidós.

Pariser, Eli (2017). El filtro burbuja. Barcelona: Taurus.

Pérez Zafrilla, Pedro Jesús (2020). Polarización política: Estado de la cuestión y orientaciones para el análisis. En Cristián Santibáñez (Ed.). Emociones, argumentación y argumentos (97-124). Lima: Palestra.

Shalabi, Ahmad S., Abdel Aal, Safaa, Abdel Halim, Wael S. y Ammar, H.Y. (2007). Artificial polarization effects on FA1:Sr2+ lasers and $\mathrm{NO}$ interactions at $\mathrm{NaCl}$ (oo1) surface: First principles calculations. Journal of Molecular Sctructure: THEOCHEM, 823, 47-58.

Schkade, David, Sunstein, Cass y Hastie, Reid (2010). When deliberation produces extremism. Critical Review, 22, 227-252.

Sunstein, Cass (2002). The law of group polarization. The Journal of Political Philosophy, 10, 175-195. 
Sunstein, Cass (2003). República.com. Internet, democracia y libertad. Barcelona: Paidós.

Sunstein, Cass (2008). Neither Hayek nor Habermas. Public Choice, 134, $87-95$.

Sunstein, Cass (2017). \#Republic: Divided democracy in the age of social media. New Jersey: Princeton University Press.

Tosi, Justin y Warmke, Brandon. (2018). Moral grandstanding. Philosophy and Public Affairs, 44, 197-217.

Yang, Jung Hwan, Hernando, Rojas, Wojcieskzak, Magdalena, Aalberg, Toril, Coen, Sharon, Curran, James, Hawasi, Kaori, Iyengar, Shanto, Jones, Paul, Mazzoleni, Gianpietro, Papathanassopoulos, Stylianos, Rhee, June W., Rowe, David, Soroka, Stuart y Tiffen, Rodney (2016). Why are «others» so polarized? Perceived political polarization and media use in ten countries. Journal of Computer-Mediated Communication, 21, 349-367.

Zuiderveen Borgesius, Frederik, Trilling, Damian, Möller, Judith, Bodó, Balázs, Vreese, Claes H. de y Helberg, Natali (2016). Should we worry about filter bubbles? Internet Policy Review, Journal of internet regulation, $5,1-16$. 